\title{
Numerical analysis on the Hydrodynamic Response of Large-scale Reclamation Project in the Hangzhou Bay
}

\author{
Wei Ronglian, Chen Wei ${ }^{a}$, Xu Tianle, Mao Chenhao, Guo Ran, Zhang \\ Mingzong and Wang Chengjie
}

School of Port and Transportation Engineering, Zhejiang Ocean University, Zhoushan 316000, China

a Corresponding email, chenwei112233@163.com

Keywords: Large scale Reclamation; Hangzhou Bay; Hydrodynamic; Mike21

Abstract.Over the past few decades, there has been large-scale land reclamation in the Hangzhou Bay, which inevitably has affected the hydrodynamics in the bay. In this study we set up a 2D hydrodynamic model based on Mike21 Model to investigate the changes of hydrodynamic in the Hangzhou Bay due to large-scale reclamation. This model is well validated with the measured data. After that the validated model is run with different coastlines in 1960s and 1985 respectively. The simulated results indicate that after the large-scale reclamation project of the Hangzhou Bay, 1): The high tide level increases and low tide level decreases, which lead to the tidal difference increase; 2): the time of the ebb turning to flood at Zhapu and HZB1 are both ahead, and the flow direction has no significant change out of the bay; 3) The flow magnitude reduces from the mouth of the bay to the inside, and the extent of reduction increases with the speed reaching $1 \mathrm{~m} / \mathrm{s}$ as it to Haiyan.

\section{Introduction}

The Hangzhou Bay (Fig.1) is located in the East China Sea, immediately south of the Changjiang Estuary. The Hangzhou Bay is a funnel-shaped, wide and shallow estuary, covering an area of approximately $8500 \mathrm{~km}^{2}$, with width to be about $100 \mathrm{~km}$ at mouth and $25 \mathrm{~km}$ at upstream reach and length of around $100 \mathrm{~km}$. The average depth of the Hangzhou Bay is 8-10 m during low tides. From the 1960s, the project "regulation and reclamation" was conducted in the Hangzhou Bay. As of 2008, in order to control the tidal prism, the reclamation area reached to 1.68 million mu. Large-scale reclamation could strongly disturb the balance of the natural hydrodynamic system, which directly affect the stability and sustainability of the seashore. Therefore, it is important to study the impact of the drastic changes in coastal configuration and evaluate the effect of the proposed reclamation plan on tidal currents.

The numerical simulation method has been widely applied to study the effect of land reclamation on the hydrodynamics in many coastal and estuarine areas. Kuang et al [1] established a 3D hydrodynamic model for the Pearl River Estuary based on Delft3D to study the changes in tidal circulation and flushing in Victoria Harbour, Hong Kong. The tidal-driven flow field and average residence time for water in Jiaozhou Bay in the years 1966, 1988, 2000, and 2008 were investigated using the EFDC with a coupled dye module [2].

This study applies Mike21 model to set up a depth-averaged hydrodynamic model in the Hangzhou Bay in order to simulate the hydrodynamical change due to large-scale reclamation. 


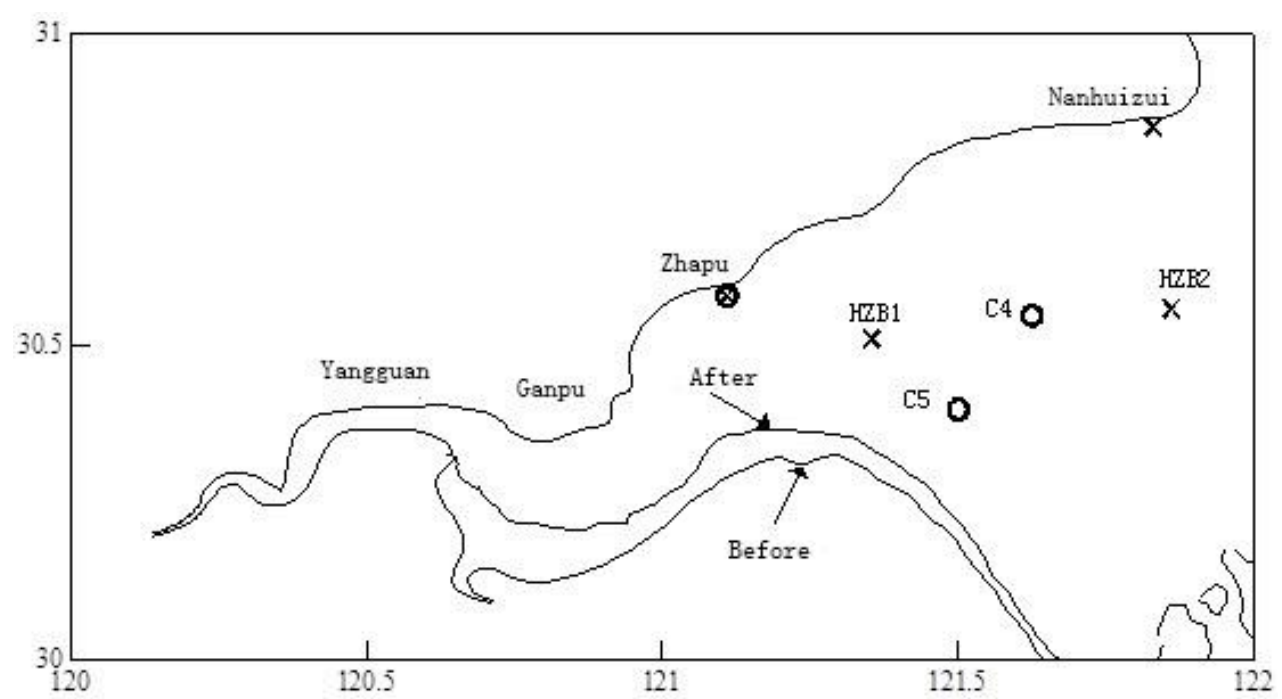

Fig.1 The Hangzhou Bay and positon of verification station and analysis points

\section{Numerical model}

A depth-averaged two-dimensional (2D) model based on Mike21 Flow Model FM finite volume model, developed by the DHI [3], is established to study the responses of Large-scale Reclamation Project in the Hangzhou Bay. Mike21 Flow Model FM is based on a flexible unstructured mesh approach and has been used for applications in oceanographic, coastal and estuarine environments. The system is based on the numerical solution of the two dimensional incompressible Reynolds averaged Navier-Stokes equations with the assumptions of Boussinesq and hydrostatic pressure. The spatial discretization of the governing equations is performed using an element-centred finite volume method. The spatial domain is discretized by subdivision of the continuum into non-overlapping elements. In the $2 \mathrm{D}$ model the elements can be triangles or quadrilateral elements. The governing equations see the manual [3].

\section{Study area and computational mesh}

The flexible unstructured triangle mesh is applicable to the regime with three bifurcations and four river outlets in the Changjiang Estuary. The spatial extent of the model is from $29^{\circ} \mathrm{N}$ to $32.5^{\circ} \mathrm{N}$ in the N-S direction and from $120^{\circ} \mathrm{E}$ to $124^{\circ} \mathrm{E}$ in the E-W direction. The Jiangyin in the Changjiang Estuary and Cangqian in the Hangzhou Bay are specified as river boundaries. The computational domain is composed of an unstructured triangular mesh with 18,006 nodes and 33,656 elements with the largest mesh at the east ocean boundary and the smallest in the North Passage. The mesh size generally decreases from the open sea to the near shore, and the finest mesh appears in the river channels.

\section{Boundary conditions and parameter settings}

The open sea boundaries are specified as time-varying tidal levels derived from a calibrated model, covering all the China Sea and adjacent seas, whose open boundary is driven by the Global Tidal Model derived from 10 years of TOPEX/POSEIDON satellite altimeter data from 1992 to 2002. The wet and dry treatment for tidal flats is introduced into this model. The Courant Friedrich Levy (CFL) condition can be used as a guide for selecting the time step, and the self-adapting time step is in a range from 0.01 to 30 seconds to keep the CFL less than 1 . The initial tidal level and velocity are defined as $1 \mathrm{~m}$ and $0 \mathrm{~m} / \mathrm{s}$ respectively. The Manning number $M$ in this study is in a range from 74 to 90 .

\section{Model verification}

The time series of hourly tidal level at Zhapu and velocity at 2 stations from June 30, 2009 to July 1, 2009 are collected to validate the simulations (Fig.2 and Fig.3). The computed values match well with the measured values. So this model can be used to simulate the hydrodynamical change due to large-scale reclamation in the Hangzhou Bay. 


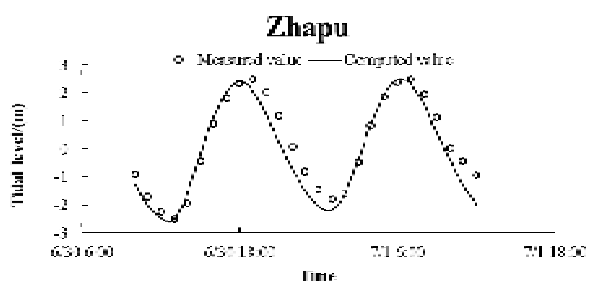

Fig. 2 Tidal level verification
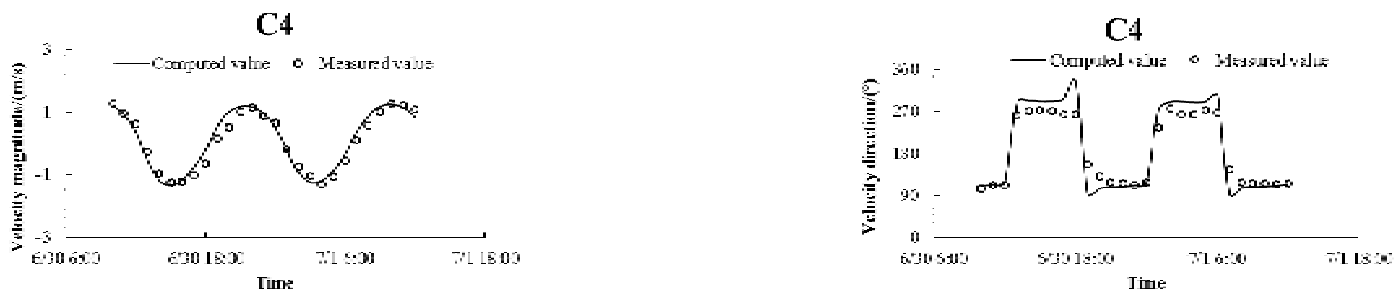

C5

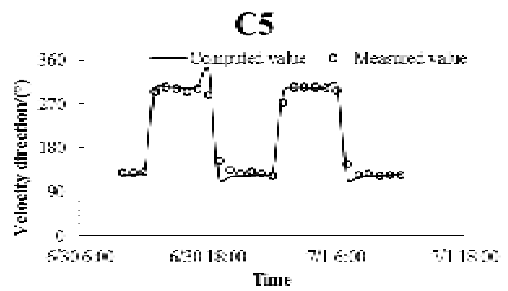

Fig.3 Flow velocity and flow direction validation

\section{Results and discussion}

The analysis points such as Nanhui, Zhapu, HZB1 and HZB2 are shown in Fig.1. Fig.5 shows the tidal level process before and after the large-scale reclamation project. After the large-scale reclamation project of the Hangzhou Bay, the high tide level increases and low tide level decreases, which lead to the tidal difference increase. The tidal range at Zhapu is larger than that at Nanhui, and the high tide level of the HZB1 is larger than that of the HZB2. The increase of tidal difference from the bay to the upstream indicates that the reclamation in the Hangzhou Bay at the south bank narrows the river channel, which makes the high tide level rise and the tidal range increase, the same conclusion as the research conclusion in document [4]. The variation of tidal level after large-scale reclamation project is shown in Fig. 5. The amplification of tidal level at Hangzhou bay from the bay mouth to the top of the bay rises gradually, which reaches to the maximum at Qibao and then gradually decreases at the upper reaches of the Hangzhou Bay. Fig.6 is a flow magnitude process before and after the large scale reclamation project, the speed at the north bank generally increases after the large-scale reclamation project. After the large-scale reclamation project, the speed amplification from Nanhuizui to Zhapu increases, besides the strength of ebb in stream central line of HZB2 outside of the bay generally increases, while the strength of flood inside of the bay slightly goes up and the strength of ebb slightly decreases. Fig.7 shows the flow direction before and after the large-scale reclamation project, which indicates that the effect of large-scale reclamation project on the flow direction in the bay is greater than that out of the bay. In addition, the time of the ebb turning to flood of Zhapu and HZB1 are both ahead, and the flow direction has no significant change out of the bay. 

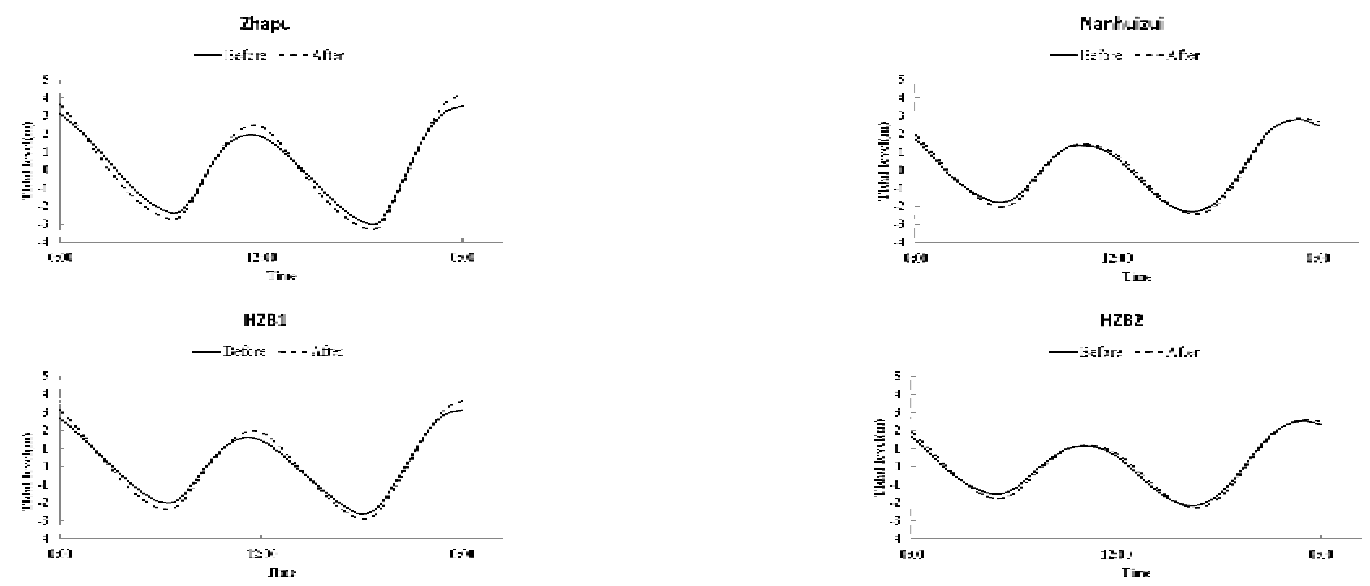

Fig.4 Tidal level process before and after the large-scale reclamation project

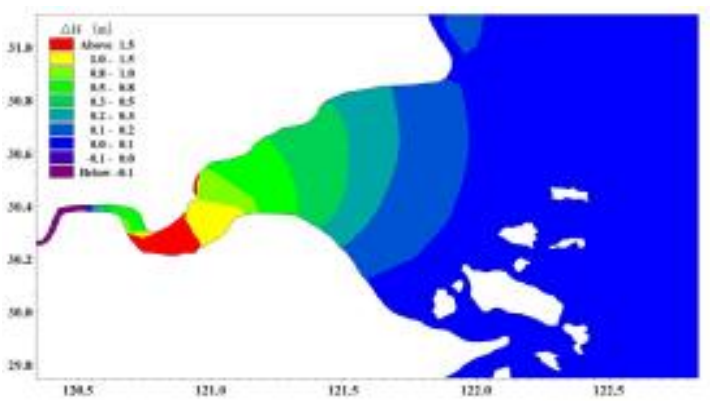

Fig.5 Change of tidal level after large-scale reclamation project (After - Before)
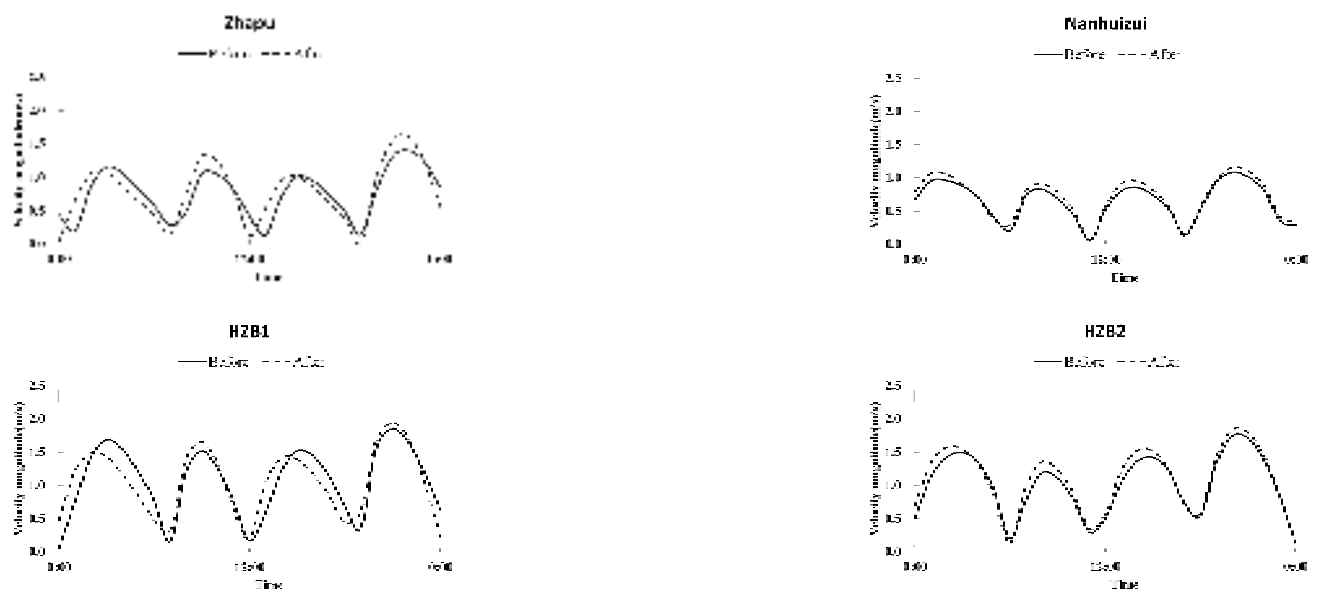

Fig.6 Flow magnitude before and after large-scale reclamation project
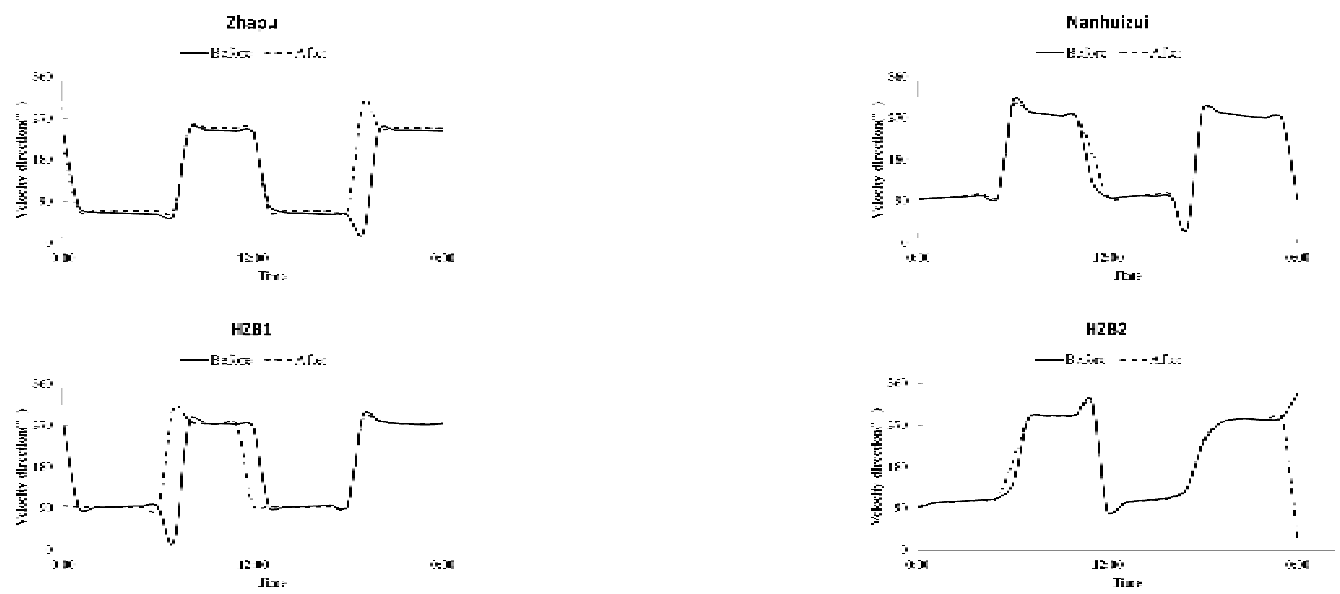

Fig.7 Flow direction before and after large-scale reclamation project 
Fig. 8 show the changes of flow magnitude after large-scale reclamation project. The flow magnitude reduces from the bay estuary to the inside, and the extent of reduction increases with the speed reaching $1 \mathrm{~m} / \mathrm{s}$ as it to Haiyan. However the river width rapidly narrows from Qibao to the upper reaches, so that flow velocity increase obviously and the maximum speed increases to $1.5 \mathrm{~m} / \mathrm{s}$.

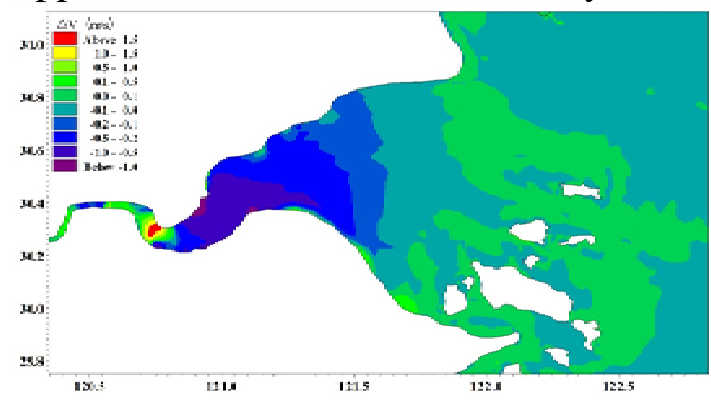

(a) change of flood tidal speed after large-scale reclamation project

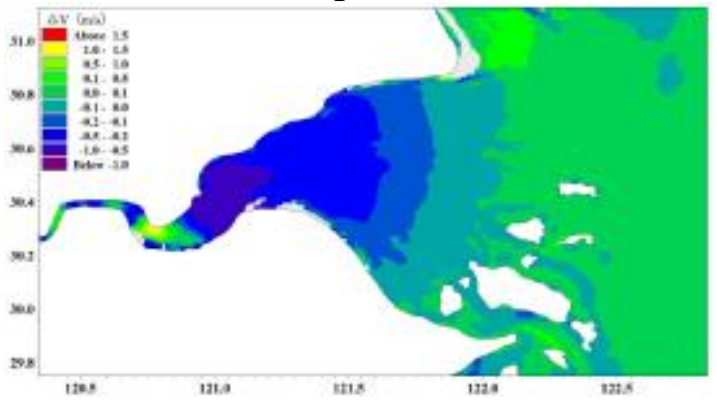

(b) change of ebb flow velocity after large-scale reclamation project

Fig.8 Changes of flow magnitude before and after large-scale reclamation project (After - Before )

\section{Conclusions}

In this paper, a 2D hydrodynamic model based on Mike21 Model is established to investigate the changes of hydrodynamic in Hangzhou Bay due to large-scale reclamation. The simulated results indicate that after the large-scale reclamation project of the Hangzhou Bay, 1): The high tide level increases and low tide level decreases, which lead to the tidal difference increase; 2 ): the time of the ebb turning to flood at Zhapu and HZB1 are both ahead, and the flow direction has no significant change out of the bay; 3) The flow magnitude reduces from the mouth of the bay to the inside, and the extent of reduction increases with the speed reaching $1 \mathrm{~m} / \mathrm{s}$ as it to Haiyan.

\section{Acknowledgements}

The work was financially supported by the STCP of Zhejiang Ocean University in 2017 and Research starting and Innovation Foundation of Zhejiang Ocean University.

\section{References}

[1] Kuang, C.P.; Huang, J.; Lee, J.H.W., and Gu, J. 2013. Impact of large-scale reclamation on hydrodynamics and flushing in Victoria Harbour, Hong Kong. Journal of Coastal Research, 29(6A), 128-143. Coconut Creek (Florida), ISSN 0749-0208.

[2] Shi, J.H.; Li, G.X., and Wang, P., 2011. Anthropogenic influences on the tidal prism and water exchanges in Jiaozhou Bay, Qingdao, China. Journal of Coastal Research, 27(1), 57-72.

[3] DHI.2011.MIKE 21\&MIKE 3 FLOW MODEL FM Hydrodynamic and Transport Module Scientific Documentation.

[4] Liu, X. 2D Numerical Simulation of the Storm Surge in the Hangzhou Bay [D]. Shanghai: Tongji University, 2012. 